\title{
Las redes sociales en la educación
}

\section{The social media in education}

\author{
Sandra A. Jaimes-Barrera ${ }^{a}$, Paola V. Ramírez-Aguilar ${ }^{b}$, Brenda M. Quijano-Luna ${ }^{c}$, Irma I. de \\ León-Vázquez ${ }^{d}$, Ivette Flores-Jiménez ${ }^{e}$, Delia I. Tapia-Castillo ${ }^{f}$
}

\begin{abstract}
:
The rise and spread of social networks has reached unthinkable levels. Thanks to the contributions of Web 2.0, social networks are gaining ground compared to other communication alternatives. A social network is a social structure composed of people, which are connected by one or several types of relationships, such as friendship, kinship, common interests, economic exchanges, or who share beliefs or knowledge. As mentioned by the author "Levis, Diego" Social networks have a huge appeal in the personal and relationship aspect on the part of the user. For this reason, the greater the number of participants, the more attraction it generates in the students. Returning to what is written by the author "Juan José del Haro" There is no doubt that information moves through technology and you have to know how to take advantage of it; where he comments that education has changed rapidly nowadays, teachers are no longer the only source of information that students can turn to since thanks to this universal access, we find students who are "experts" in certain areas of knowledge that are of interest to them and are able to learn autonomously. The author "Irene Garrigós" tells us that social networks and the use of technology in teaching are on the rise since they are very useful creating closer communication between students and the teacher. Artero (2011) says that the management of a social network in the classroom can lead to a rapprochement between the teacher and the student. Teachers are no longer communicators or transmitters of knowledge to be tutors who guide students through the educational process trying to enhance their participation.
\end{abstract}

\section{Keywords:}

Social networks, education, teachers, students

\section{Resumen:}

El auge y la propagación de las redes sociales han alcanzado niveles impensados. Gracias a los aportes de la Web 2.0 las redes sociales están ganando terreno frente a otras alternativas de comunicación. Una red social es una estructura social compuesta de personas, las cuales están conectadas por uno o varios tipos de relaciones, tales como amistad, parentesco, intereses comunes, intercambios económicos, o que comparten creencias o conocimiento. Como lo menciona el autor "Levis, Diego" Las redes sociales tienen un enorme atractivo en el aspecto personal y de relación por parte del que las usa. Por este motivo, cuanto mayor sea el número de los participantes más atracción genera en los alumnos. Retomando lo escrito por el autor "Juan José del Haro" Es indudable que la información se mueve a través de la tecnología y hay que saber sacarle provecho; en donde comenta que la educación ha cambiado de manera rápida en la actualidad, los maestros ya no son la única fuente de información a la que los alumnos pueden acudir ya que gracias a este acceso universal, encontramos alumnos que son "expertos" en determinadas áreas del conocimiento que son de su interés y son capaces de aprender de forma autónoma. El autor "Irene Garrigós" nos dice que las redes sociales y el uso de la tecnología

\footnotetext{
a Autor de Correspondencia, Universidad Autónoma del Estado de Hidalgo, Escuela Superior de Tlahuelilpan, https://orcid.org/0000-00016968-0567, Email: ja381309@uaeh.edu.mx

${ }^{\text {b }}$ Universidad Autónoma del Estado de Hidalgo, Escuela Superior de Tlahuelilpan, https://orcid.org/0000-0002-8747-2005, Email: ra413346@uaeh.edu.mx
}

c Universidad Autónoma del Estado de Hidalgo, Escuela Superior de Tlahuelilpan, https://orcid.org/0000-0001-6115-6306, Email: qu304313@uaeh.edu.mx

d Universidad Autónoma del Estado de Hidalgo, Escuela Superior de Tlahuelilpan, https://orcid.org//0000-0001-9197-0050, Email: irmalv@uaeh.edu.mx

e Universidad Autónoma del Estado de Hidalgo, Escuela Superior de Tlahuelilpan, https://orcid.org/0000-0002-9591-4416, Email: ivette@uaeh.edu.mx

f Universidad Autónoma del Estado de Hidalgo, Escuela Superior de Tlahuelilpan, https://orcid.org/0000-0001-7522-543X, Email: itapia@uaeh.edu.mx 
en la docencia va en auge ya que estas son muy útiles creando una comunicación más estrecha entre los alumnos y el profesor. Artero (2011) dice que en el manejo de una red social en el aula puede suponer un acercamiento entre el docente y el alumno. Los profesores dejan de ser comunicadores o transmisores de conocimiento para ser tutores que guían a los alumnos a través del proceso educativo intentando potenciar la participación de los mismos.

\section{Palabras Clave:}

Redes sociales, educación, docentes, alumnos

\section{Introducción}

Para comenzar se explicará qué es una red social "La red social es una plataforma digital de comunicación global que pone en contacto a gran número de usuarios" según la Real Academia Española.

Para hablar sobre las redes sociales es conveniente abordar dos términos, la World Wide Web y la Web 2.0. La web en su forma original proporcionaba información, pero no facilitaba la participación del usuario.

Abordando la historia de las redes sociales, con base en Ponce (2012), se tiene lo siguiente: 1971. Se envía el primer correo electrónico entre dos computadoras, una al lado de la otra. 1995. La web alcanza el millón de sitios, y The Globe ofrece a los usuarios la posibilidad de personalizar sus experiencias en línea, mediante la publicación de su propio contenido y conectando con otros individuos de intereses similares. El mismo año, Randy Conrads crea Classmates, una red social para contactar con antiguos compañeros. Classmates es para muchos el primer servicio de red social. 1997. Se lanza $A O L$ Instant Messenger. Se inaugura Sixdegrees, red social que permite la creación de perfiles personales y listado de amigos. 2003. Nacen MySpace, Linkedln y Facebook, Creada por Mark Zuckerberg, Facebook se concibe inicialmente como una plataforma para conectar a los estudiantes de la Universidad de Harvard. 2006. Se inaugura la red social de microblogging Twitter. Google cuenta con 400 millones de búsquedas por día. 2010. Google lanza Google Buzz, su propia red social integrada con Gmail, en su primera semana sus usuarios publicaron nueve millones de entradas.

Las redes sociales se han introducido en el sistema educativo y en la actualidad existen varias plataformas que se han orientado hacia el ámbito educativo.

Edmodo.com: Ofrece a los profesores y estudiantes un lugar seguro para colaborar, compartir contenidos y aplicaciones educativas, discusiones y notificaciones. Tienen por objetivo ayudar a los docentes a aprovechar el potencial de los medios sociales para personalizar el aula para cada estudiante.

Eduredes.ning.com: Es una red gratuita, cuya finalidad es la de actuar como punto de encuentro entre docentes y personas interesadas en la enseñanza y aprendizaje a través de las redes sociales.
GoingOn.com: Ofrece una solución bajo demanda para la construcción de redes sociales académicas privadas.

\section{Importancia}

Las redes sociales son una herramienta que ayuda al aprendizaje colaborativo entre alumnos y profesores. Su uso les permite a los profesores enseñar a los estudiantes a adquirir capacidades para valerse por sí solos, para así aprender sobre el proceso acelerado de cambios y transformaciones en la que estamos sometidos por las nuevas tecnologías. Ofrecen la integración de herramientas como blogs, chats, foros, video conferencias, las cuales resultan eficientes para la enseñanza y el aprendizaje.

No solo permiten la transmisión de conocimientos, sino que, además, desarrollan competencias tecnológicas, hacen posible que los alumnos desarrollen habilidades y aptitudes, como lo es el trabajo en equipo, los procesos sociales de participación, el razonamiento, la capacidad de análisis y la toma de decisiones que caracterizan a una sociedad avanzada y democrática.

Contar hoy con una red social educativa es imprescindible, además de impartir conocimiento a los alumnos, también se usan para compartir hallazgos científicos e impedir que el conocimiento nuevo quede estático, es decir, se trabaja por la democratización del conocimiento y su difusión.

\section{Beneficios}

Las redes sociales educativas en nuestra sociedad forman parte de nuestra vida y cultura ya que mejora la comunicación tanto dentro de clase como fuera. Al estar profesores y alumnos en la misma red social hace que fluya la comunicación. Sin embargo, se encuentran algunas dificultades ya que a los alumnos se les hace un poco complicado el uso de las plataformas y más cuando no se tiene un entendimiento de ellas. También es una forma que pueden tener los profesores de conocer mejor a sus alumnos, sus inquietudes, necesidades 0 problemas.

Para los profesores también es una oportunidad para el aprendizaje, muchos de los educadores tendrán que formarse en este nuevo mundo de las redes sociales 
educativas y son una oportunidad para adquirir nuevos conocimientos y habilidades.

\section{Ventajas y Desventajas}

\begin{tabular}{|c|c|}
\hline Ventajas & Desventajas \\
\hline $\begin{array}{l}\text { - Genera competencias } \\
\text { tecnológicas en el } \\
\text { alumno. } \\
\text { - Fomenta la } \\
\text { investigación. } \\
\text { - } \quad \text { Trabajo en equipo. } \\
\text { - Creación de una } \\
\text { identidad digital. } \\
\text { - Acceso a una clase } \\
\text { invertida. } \\
\text { - Creatividad digital. }\end{array}$ & $\begin{array}{l}\text { Distracciones } \\
\text { constantes. } \\
\text { - Reducción de las } \\
\text { relaciones humanas. } \\
\text { - Son un complemento } \\
\text { de aprendizaje. }\end{array}$ \\
\hline
\end{tabular}

Estas ventajas como el generar competencias tecnológicas, será una herramienta fundamental en el campo laboral; el fomentar la investigación creará nuevas oportunidades de mejorar lo que ya existe; el trabajo en equipo como ventaja es una forma de trabajo que ahora se realizará a distancia y deben estar familiarizados los estudiantes; el hecho de contar con una identidad digital permitirá que diferentes empleadores conozcan el trabajo de los estudiantes y conozcan el perfil en que se manejan para el reclutamientos de personal; el tener el acceso a clases desde donde se encuentren o al trabajo será otra modalidad que sólo aquellos que se atrevan a utilizar las redes podrán llevar a cabo esta actividad u por último la creatividad digital, al realizar las actividades apoyados en diferentes plataforma permitirá que sea más rápido y dejará espacio a lo creativo.

En lo que corresponde a las desventajas no se pueden controlar los distractores, pero se darán soluciones como la aparición de cafés-internet, donde las personas contarán con wifi a buena velocidad, trabajarán sin distracciones y podrán tomar un rico café; la reducción de las relaciones humanas, es difícil considerar que no existan pues por naturaleza el hombre necesarita de sus círculos de amigos y por último se considera una desventaja por ser sólo un complemento del aprendizaje, sin embargo, cada vez será más frecuente trabajar a través de plataformas y redes sociales.

\section{Comentarios}

Las redes sociales influyen en la educación son una herramienta fundamental, permite agilizar la educación, ha cambiado la forma de comunicarnos, ha cambiado la forma que los alumnos pueden aprender, pueden considerarse educativas, fomentan el dialogo y la participación de los alumnos, ayuda a desarrollar competencias digitales y tecnológicas.

Los alumnos deciden cómo y en qué orden desea acceder a la amplia base de datos con las que cuenta. Sin embargo, la velocidad del aprendizaje de los usos para los nuevos medios no ha evolucionado con la misma rapidez que el desarrollo de contenidos.

Por ello es importante que los usuarios (alumnos, padres de familia y profesores), consideren importante la inversión en un buen servicio de internet, en una computadora o un móvil que les permita tener acceso a las plataformas educativas y a sus actualizaciones.

Se podría pensar que sólo los que tienen recursos económicos tendrán acceso a la educación, pero no se trata del costo se trata de la oportunidad que se tendrá de poder participar en un mundo diferente al que conocemos actualmente. Todas las familias hacen el esfuerzo por la compra de uniformes, de libros, de mochilas, de pago de cuotas o colegiaturas; lo que va a cambiar es todo lo anterior por un servicio de internet, computadora o móvil. Siguen siendo herramientas para la educación.

\section{Conclusiones}

Para concluir las redes sociales se están convirtiendo en una herramienta que permite el desarrollo de los estudiantes, pues la mayoría de ellos las usan, las incluyen como parte de sus actividades académicas.

Las redes sociales se conciben desde y para la interacción y este nuevo espacio de diálogo puede ser un lugar de aprendizaje y enriquecimiento mutuo, el docente interviene, modula y colabora en el aprendizaje con su alumnado. Considerando lo anterior, las redes crean un sitio de interacción, individualidad y multiculturalidad y, al mismo tiempo, enriquecen el aprendizaje colaborativo.

Pero debemos ser conscientes de que se puede tener resistencia al cambio, donde algunos alumnos o docentes no aceptarán esta nueva modalidad de enseñanzaaprendizaje, queriendo tener un retroceso a lo cotidiano y no querer avanzar. Existirán elementos de la educación como son los alumnos, padres de familia o docentes que buscarán boicotear este avance de la tecnología en la educación, sin embargo, el progreso no se puede detener y será necesario que busquen incorporarse a esta nueva forma de trabajar que no tiene paso atrás o quedarán en una era de la educación que solo será un recuerdo, pues junto con la educación cambiarán las formas de trabajar o buscar trabajo.

\section{Referencias}


[1] R. V. Argüelles, «Revista Digital Universitaria,» 1 Abril 2013. [En línea]. Available: http://www.revista.unam.mx/vol.14/num4/art36/index.html.

[2] L. Castellanos, «Tecnología al día,» 21 Junio 2014. [En línea]. Available: https://tecnologiaaldia.wordpress.com/2014/06/21/importancia-delas-redes-sociales-en-la-educacion/.

[3] E. Miranda, «MEDAC,» 11 Septiembre 2018. [En línea]. Available: https://medac.es/articulos-educacion-infantil/influyen-las-redessociales-la-educacion/.

[4] F. Naso, M. L. D. G. N. Balbi y J. A. Peri, «La importancia de las Redes sociales en al ámbito educativo,» [En línea]. Available: http://sedici.unlp.edu.ar/bitstream/handle/10915/18296/Documento_ completo_.pdf?sequence=1.

[5] M. Vázquez-Reina, «Consumer,» 2 Febrero 2011. [En línea]. Available: https://www.consumer.es/educacion/juan-jose-de-harodoctor-en-biologia-y-autor-del-libro-redes-sociales-para-laeducacion.html.

[6] «Redem,» 1 Diciembre 2017. [En línea]. Available: https://www.redem.org/educacion-3-0-usos-de-las-redes-sociales-enel-aula-ventajas-y-desventajas/.

[7] S. Rodríguez, «Emagister,» [En línea]. Available: https://www.emagister.com/blog/la-importancia-de-las-redessociales-en-la-educacion-actual/.

[8] C. I. Torres, «Universidad de Guadalajara,» 2011. [En línea]. Available: http://www.udgvirtual.udg.mx/apertura/index.php/apertura/article/vi ew/198/213. 